\title{
HONGOS ASCOMICETOS DEL BOSQUE MESÓFILO DE MONTAÑA EN MÉXICO
}

\author{
Rosario Medel \\ Universidad Veracruzana, Instituto de Investigaciones Forestales, \\ Apdo. postal 551, 91070 Xalapa, Veracruz, México. \\ romedel@uv.mx
}

\section{RESUMEN}

Se presenta una puesta al día de la diversidad de los ascomicetos citados del bosque mesófilo de montaña (BMM) en México. A la fecha se conocen un total de 107 especies de las cuales 69 están presentes en el estado de Veracruz. Los taxa corresponden a 18 órdenes, 29 familias y 52 géneros. La familia Xylariaceae es la más diversa, y los géneros Xylaria e Hypoxylon los mejor representados. Algunas de las características ecológicas de las especies fueron analizadas; se encontró que el grupo trófico de hongos saprobios es el más abundante, mientras que la madera en descomposición es el sustrato donde se encontraron la mayoría de las especies. En cuanto a la diversidad taxonómica de los hospederos, 20 familias y 22 géneros de plantas vasculares se registraron en asociación con este grupo de hongos. Al menos una familia de ascomicetos registrada del bosque mesófilo (Hyaloscyphaceae) podría considerarse recurrente en helechos de la familia Cyatheaceae.

Palabras clave: bosque de niebla, hongos en helechos, hongos lignícolas, hongos saprobios, Hypoxylon, Xylaria.

\section{ABSTRACT}

An update of the species of Ascomycetes recorded for cloud forest in Mexico is presented. Currently, 107 species of Ascomycetes have been recorded in this type of vegetation, of which 69 are present in Veracruz. The taxa correspond to 18 orders, 29 families, and 52 genera. The Xylariaceae is the most diverse family, and Xylaria and Hypoxylon are the genera with the highest number of species. Some of the ecological characteristics of the species were surveyed; it was found that the trophic group of the saprobes is the 
most diverse, while decaying wood is the substrate where most of the species were found. Regarding the taxonomic diversity of the hosts, 20 families and 22 genera of vascular plants were recorded in association with this group of fungi. At least one family of the fungi recorded in cloud forest (Hyaloscyphaceae) could be considered as recurrent on ferns of the family Cyatheaceae.

Key words: cloud forest, fungi on ferns, Hypoxylon, lignicolous fungi, saprobe fungi, Xylaria.

\section{INTRODUCCIÓN}

La distribución de la diversidad de algunos grupos de organismos en diversos ecosistemas del país no se conoce de manera homogénea. A pesar de los esfuerzos realizados por investigadores mexicanos y extranjeros, algunos bosques no han sido explorados del todo, sea por su distribución dispersa o su difícil acceso. Un ejemplo es el bosque mesófilo de montaña (BMM), que se encuentra en situación vulnerable debido a la superficie reducida en que prospera y a la fragmentación provocada por el hombre (Rzedowski, 1978; Challenger, 1998; Bubbs et al., 2004; Williams-Linera, 2007; Challenger y Soberón, 2008). Este bosque cubre apenas 1\% del territorio de la República Mexicana (Rzedowski, 1978, 1996; Challenger, 1998), es el más diverso por unidad de superficie (Williams-Linera et al., 2002), contiene $10 \%$ de la flora del país (Williams-Linera, 2007) y en él se encuentran grupos muy bien representados como los helechos con 500 especies citadas (Williams-Linera, 2007).

Desde el punto de vista micológico, el único trabajo que a la fecha registra la diversidad fúngica del BMM en México fue realizado por Chacón y Medel (1993). Estos autores citaron 594 especies de hongos, de las cuales solo 96 son ascomicetos; sin embargo, muchas de las que mencionan también prosperan en otros tipos de vegetación, como el bosque de coníferas, el bosque de encinos o los bosques tropicales en sus diferentes clases. Desde la publicación del trabajo en 1993, se ha avanzado en el estudio de los ascomicetos mexicanos y en los dos últimos inventarios (Medel et al., 1999; Medel, 2007a) se estimó que el estado de Veracruz es el que mayor número de especies registró y que el BMM es el segundo tipo de vegetación en el país con más registros de este grupo de hongos, solo superado por el conjunto de bosques tropicales (Medel, 2007a).

El objetivo de este trabajo es presentar una lista de especies de ascomicetos citadas del BMM en México, seguida de un análisis taxonómico, ecológico y de dis- 
tribución geográfica; además de documentar algunos hospederos asociados a este grupo de hongos. También se comentan algunas necesidades que sería importante cubrir para conocer de manera más certera la repartición de las especies de ascomicetos en este tipo de vegetación.

\section{MATERIALES Y MÉTODOS}

Este análisis incluyó la revisión de los trabajos micológicos que han citado especies de ascomicetos del BMM en México hasta el año 2010. Se realizó una búsqueda bibliográfica de los datos de su hábitat para corroborar si se mencionaba el tipo de vegetación, hospedero o sustrato sobre el cual se encontraron. Cuando la información publicada estaba incompleta, y los especímenes podían consultarse en XAL, se completaron los datos faltantes, especialmente para algunas especies parásitas para las que se registraba el hospedero pero no se proporcionaba el tipo de vegetación donde se colectó. En algunos casos se obtuvo guía complementaria a partir de mapas de distribución del BMM, cuando en la cita solo se mencionaba la localidad, sin mayor indicación ecológica. En cuanto a la taxonomía, se consultó el Index fungorum (http://www.indexfungorum.org/names) con el objeto de actualizar los nombres de las especies. Con esta información se elaboró un cuadro en el cual se resumieron, además de las categorías taxonómicas, los datos de adscripción a grupos tróficos y sustratos. Estos últimos se asignaron con base en la información bibliográfica o de las etiquetas del material de herbario encontrado. El carácter de simbionte micorrizógeno se acepta de acuerdo con la propuesta de Rinaldi et al. (2008).

\section{RESULTADOS}

La revisión de ejemplares de herbario y bibliográfica dio por resultado el reconocimiento de 107 especies de ascomicetos para el BMM en México (Apéndice). Para cada una se anotó el orden y la familia a la que está adscrita, la entidad federativa de la cual se conoce, el sustrato donde crece, el grupo trófico y las referencias bibliográficas correspondientes. Del estado de Veracruz se registraron 69 que corresponden a $63.3 \%$ del total de las citadas, siguiéndole Tamaulipas (con 18) y Morelos (con 9). Taxonómicamente estos hongos se agrupan en 18 órdenes, 29 familias y 52 géneros. El orden Xylariales fue el que contó con mayor representación, pues 
incluye 42 especies, siendo los géneros Xylaria con 18 e Hypoxylon con 10 los más cuantiosos. El siguiente orden de mayor diversidad fue el de los Helotiales con 18, en el cual el género Lachnum con 8 especies fue el más numeroso. En el orden Pezizales los géneros Plectania y Helvella quedaron registrados con tres cada uno (Fig. 1).

Para el tipo de sustrato sobre el que se encontraron creciendo los hongos, se determinaron ocho diferentes categorías (Fig. 2), siendo el tipo lignícola al que pertenece la mayoría de las especies (64.4\%). En segundo lugar están las folícolas $(14.9 \%)$, le siguen las terrícolas con $8.4 \%$ y con el mismo porcentaje $(2.8 \%)$ las asociadas a insectos, frutos de árboles y humícolas; la menor proporción la constituyeron los hongos fimícolas y fungícolas con 2 especies (1.8\%) cada uno.

En la misma figura 2 se pueden consultar los grupos tróficos a los que pertenecen los hongos citados, de donde se desprende que la mayoría resultaron ser saprobios (70\%), los parásitos (débiles o facultativos) constituyeron $24.2 \%$, y los simbiontes micorrizógenos ocuparon solo $5.6 \%$ del total; entre estos últimos se pueden mencionar los géneros Helvella, Humaria y Peziza.

De acuerdo con la literatura consultada (Cuadro 1), son 20 las familias y 22 los géneros de plantas del BMM de México, en las que se han encontrado creciendo ascomicetos. Las especies de Alchemilla, Hyptis, Setaria, Solanum, Crossopetalum

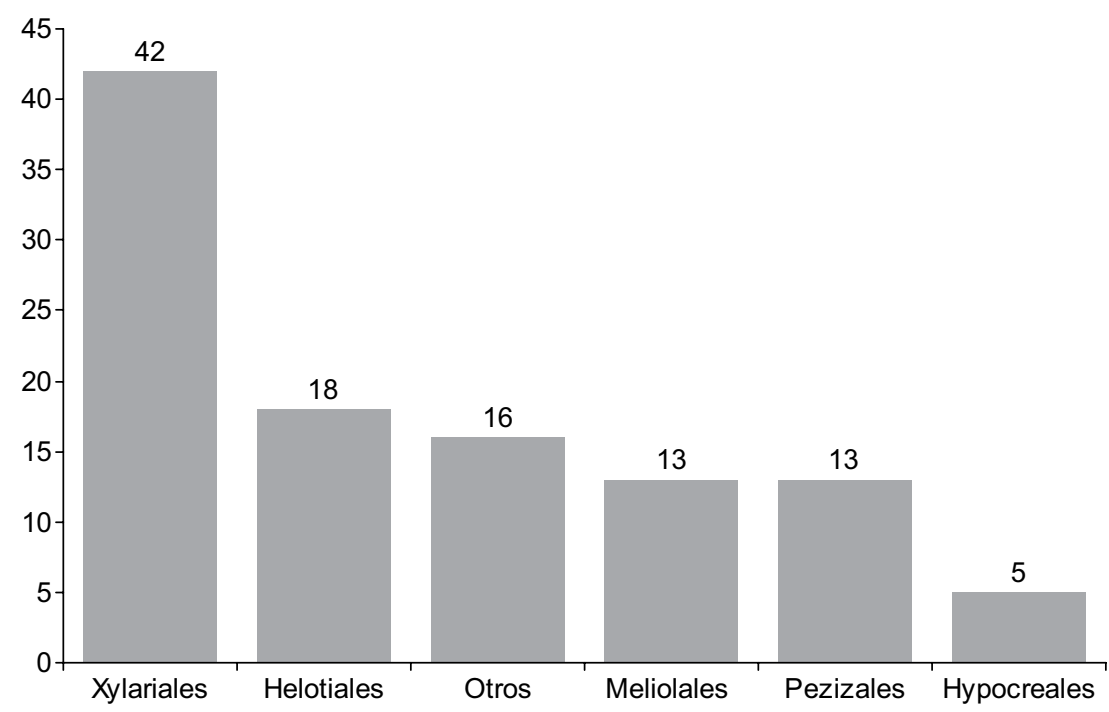

Fig. 1. Número de especies pertenecientes a los diferentes órdenes de de Ascomycetes del BMM. 


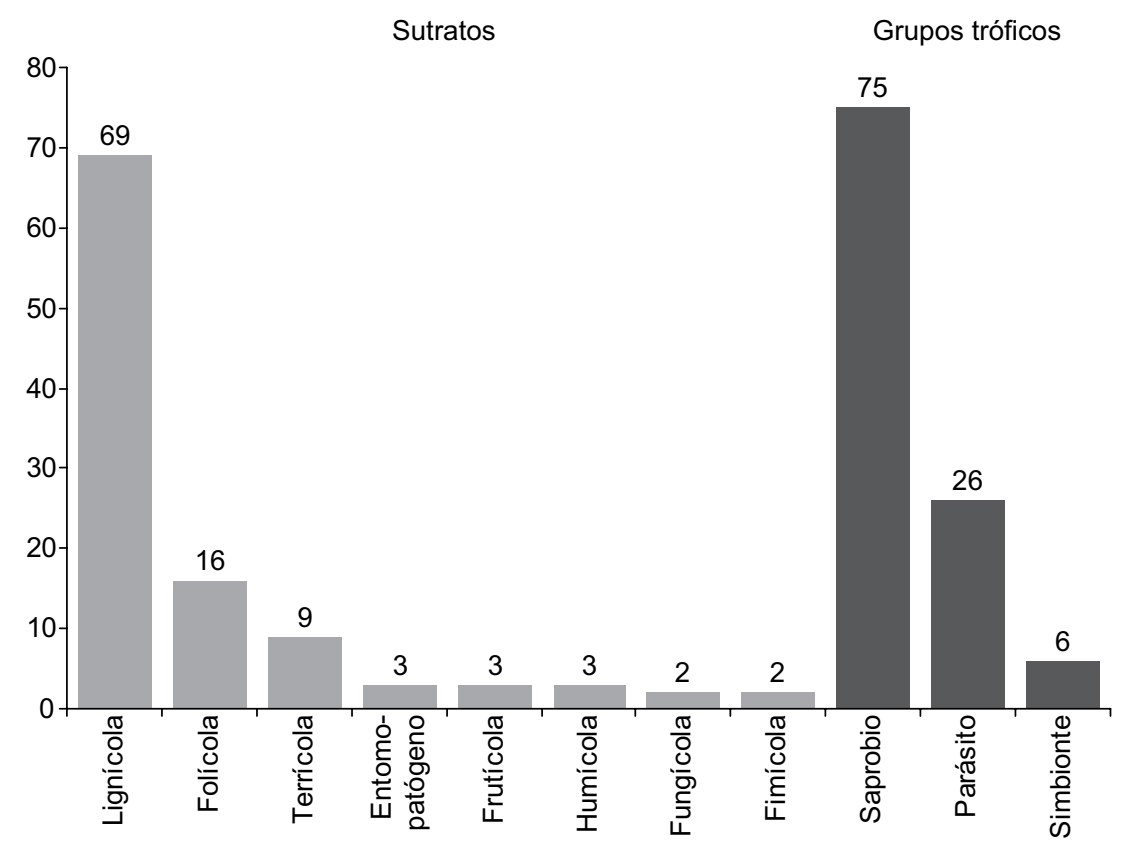

Fig. 2. Número de especies de Ascomycetes correspondientes a los diferentes sustratos y grupos tróficos.

y Rubus no son propiamente típicas o comunes en el bosque mesófilo conservado, encontrándose muchas veces de manera marginal o en la vegetación secundaria derivada (Standley y Williams, 1973; Calderón de Rzedowski, 1979; Nee, 1993; Pohl, 1994). Las de otros géneros sí son característicos del BMM, como los helechos arborescentes de los géneros Alsophila y Cyathea o las de Liquidambar, Magnolia y Platanus entre las angiospermas. De acuerdo con Rzedowski (1996), la familia Cyatheaceae es considerada casi exclusiva de este tipo de vegetación. Recientemente Medel y Lorea-Hernández (2008) y anteriormente Haines (1980) citaron varias especies de Lachnum (Fig. 3) habitando helechos arborescentes de México. Otro de los géneros comunes en este bosque es Plectania (Fig. 4) que se caracteriza por los ascomas de color negro brillante, creciendo sobre ramas de Quercus spp., tiradas en el suelo.

Existen muchas especies de Xylaria citadas de BMM; sin embargo, como lo hicieron notar San Martín y Rogers (1995), la mayoría de ellas prosperan en hospederos en avanzado estado de descomposición que son difíciles de determinar. 
Cuadro 1. Géneros de Ascomycetes asociados a familias y géneros de plantas del BMM.

\begin{tabular}{|c|c|c|}
\hline Género de ascomycete & Familia de plantas* & Género \\
\hline Lachnum & Altingiaceae & Liquidambar \\
\hline \multicolumn{3}{|l|}{ Pseudocersopora } \\
\hline \multicolumn{3}{|l|}{ Xylaria } \\
\hline Gnomonia & Betulaceae & Carpinus \\
\hline Meliola & Celastraceae & *Crossopetalum \\
\hline Meliola & Convolvulaceae & Ipomoea \\
\hline \multirow[t]{2}{*}{ Lachnum } & Cyatheaceae & Alsophila \\
\hline & & Cyathea \\
\hline Lachnum & Blechnaceae & Blechnum \\
\hline Lachnum & Marattiaceae & Marattia \\
\hline Lachnum & Thelypteridaceae & Thelypteris \\
\hline Meliola & Fabaceae & Mimosa \\
\hline Byssosphaeria & Fagaceae & Quercus \\
\hline \multicolumn{3}{|l|}{ Hymenoscyphus } \\
\hline \multicolumn{3}{|l|}{ Irenopsis } \\
\hline \multicolumn{3}{|l|}{ Mollisia } \\
\hline \multicolumn{3}{|l|}{ Plectania } \\
\hline \multicolumn{3}{|l|}{ Propolomyces } \\
\hline \multicolumn{3}{|l|}{ Sphaerotheca } \\
\hline \multicolumn{3}{|l|}{ Xylaria } \\
\hline Meliola & Gramineae & *Setaria \\
\hline Meliola & Lamiaceae & *Hyptis \\
\hline Hymenoscyphus & Magnoliaceae & Magnolia \\
\hline \multicolumn{3}{|l|}{ Xylaria } \\
\hline Meliola & Meliaceae & Trichilia \\
\hline \multirow{2}{*}{\multicolumn{3}{|c|}{ Meliola }} \\
\hline & & \\
\hline Xylaria & Myrtaceae & Eugenia \\
\hline Meliola & Piperaceae & Piper \\
\hline Hymenoscyphus & Platanaceae & Platanus \\
\hline \multicolumn{3}{|l|}{ Lachnum } \\
\hline \multirow[t]{2}{*}{ Meliola } & Rosaceae & *Alchemilla \\
\hline & & *Rubus \\
\hline Meliola & Solanaceae & *Solanum \\
\hline
\end{tabular}

*Géneros de los que varias de sus especies crecen en vegetación secundaria o en zonas marginales (ecotonos) del BMM. 

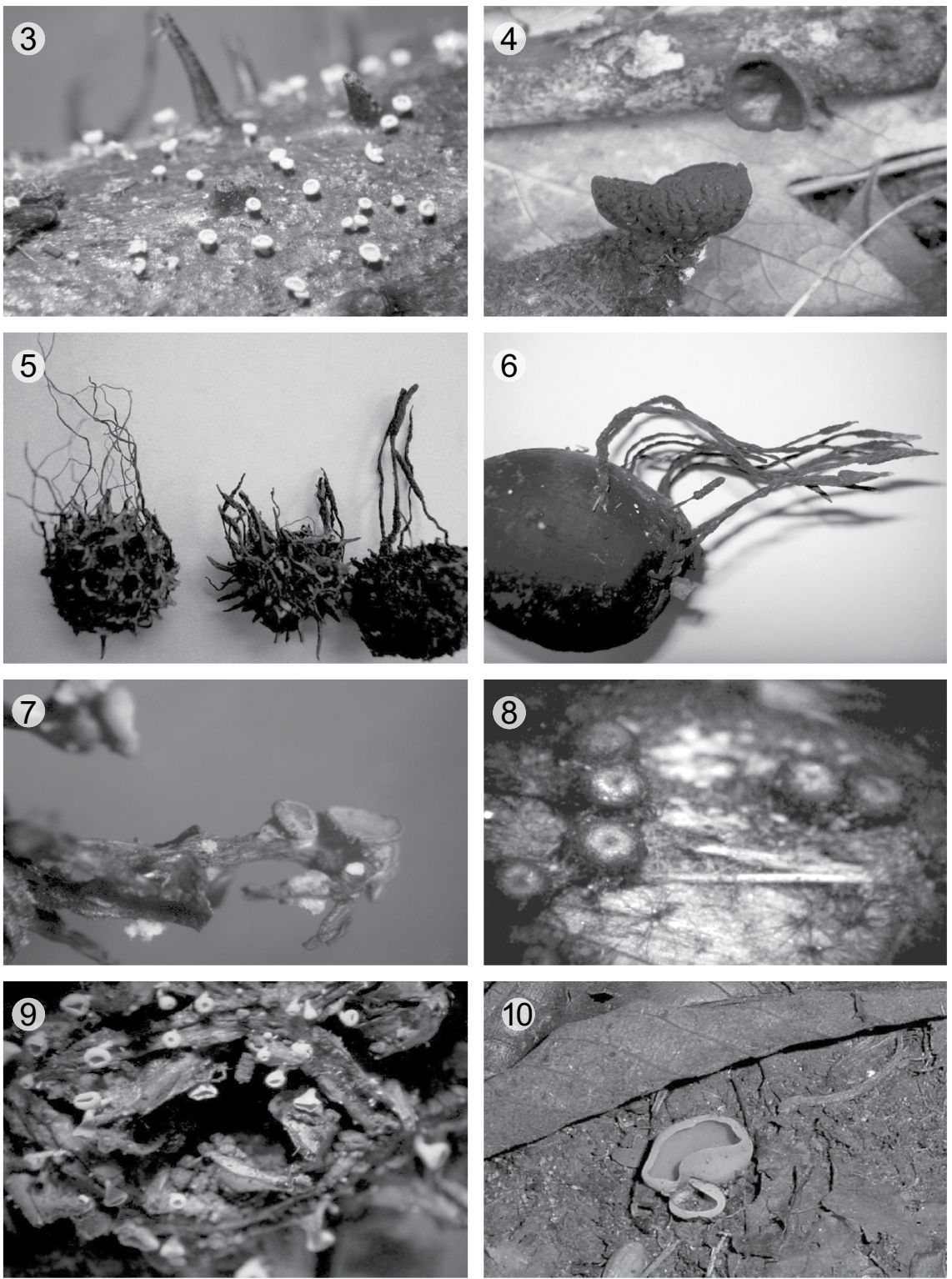

Figs. 3-10. 3. Lachnum fimbriiferum creciendo en un helecho arborescente del género Cyathea. 4. Plectania platensis, en ramas de Quercus. 5. Xylaria liquidambaris, sobre frutos de Liquidambar. 6. X. arbuscula, creciendo en frutos de Quercus. 7. Patinellaria cubensis, una especie rara del BMM, creciendo sobre estromas de X. arbuscula. 8. Byssosphaeria jamicana, sobre frutos de Quercus. 9. Hymenoscyphus fructigenus sobre frutos de Liquidambar.10. Peziza succosella especie común en el BMM del centro de Veracruz. 
En el caso de aquellas que crecen en frutos, la identificación es más fácil, siempre y cuando éstos no estén muy deteriorados. Tal es el caso de Xylaria liquidambaris (Fig. 5) que habita frutos de Liquidambar styraciflua; de Xylaria magnoliae en los de Magnolia dealbata; o de especies que viven en las hojas y frutos de Quercus spp., como X. arbuscula (Fig. 6). Mención aparte merece la presencia del discomiceto Patinellaria cubensis (Fig. 7) que crece sobre estromas de X. arbuscula, ya que este hongo a nivel mundial ha sido citado en raras ocasiones y se encontró en BMM (Medel y Chacón, 1988a). Otro de los Ascomycetes poco frecuentes es Byssosphaeria jamaicana (Fig. 8), que habita en frutos de encino. Algunos hongos del orden Helotiales son más o menos comunes en las localidades registradas, como Hymenoscyphus fructigenus (Fig. 9) en frutos de Platanus, Magnolia y Quercus. Dentro de los Pezizales, la especie terrícola Peziza succosella (Fig. 10) es muy abundante en los meses de mayo a agosto en el BMM del centro de Veracruz.

\section{DISCUSIÓN}

De las 107 especies citadas en este trabajo, solamente ocho (Cuadro 2) fueron descritas del BMM de nuestro país. De éstas, Morchella rufobrunnea se ha citado de México, Oregon y California (Guzmán y Tapia, 1998; Kuo, 2008). Las siete restantes solo se conocen de las localidades de donde fueron descritas. Aunque este estudio incluyó todas las citas para el territorio de la República, se contó con más información detallada de la parte central del estado de Veracruz, región que la autora ha explorado con mayor esfuerzo desde 1988. Concretamente las fracciones de BMM más colectadas son: Jardín Botánico Francisco J. Clavijero y Parque Ecológico El Haya, del municipio de Xalapa; paraje El Chivizcoyo en la Congregación Los Capulines y Rancho Agüita Fría, municipio de San Andrés Tlalnelhuayocán; Campestre San Rafael, región de Zoncuantla, municipio de Coatepec; y Granja Santa Bárbara, municipio de Rafael Lucio. Algunas de estas localidades corresponden a fragmentos de BMM conservado, presentes en la región oeste de Xalapa (Williams-Linera et al., 2002).

Hyde et al. (2007) caracterizaron varios niveles de asociación de hongos saprobios-hospedero. Cuando reiteradamente se hallaron las mismas especies de hongos asociados a las mismas estructuras del hospedero, denominaron "recurrente" a este tipo de relación. Con base en el análisis de la información obtenida en este trabajo, se puede inferir cierta "recurrencia" (sensu Hyde et al., 2007) en algunos casos de asociación hongo-hospedero, como en el género Lachnum de la familia Hyaloscyphaceae, que se consideraría recurrente en el raquis foliar de Alsophila fir- 
Cuadro 2. Especies de Ascomycetes descritas del BMM de México.

\begin{tabular}{lll}
\hline Especies & Estado & Referencia \\
\hline Crocicreas sessilis & Oaxaca & Samuels y Rogerson, 1990 \\
Dimeriella polypodii & Oaxaca & Samuels y Rogerson, 1990 \\
Morchella rufobrunnea & Veracruz & Guzmán y Tapia, 1998 \\
Xylaria eugeniae & Tamaulipas & San Martín et al., 1997 \\
X. jaliscoensis & Jalisco & Rogers et al., 2002 \\
X. michoacana & Michoacán & San Martín et al., 2001 \\
X. subcoccophora & Tamaulipas & San Martín et al., 2001 \\
X. tumulosa & Chiapas & San Martín et al., 2001 \\
\hline
\end{tabular}

ma y Cyathea bicrenata (ambos helechos arborescentes de la familia Cyatheaceae, típicos del BMM), y en Xylaria liquidambaris o $X$. magnoliae recurrentes en frutos de Liquidambar styraciflua y Magnolia dealbata. Por su parte, las especies parásitas de los géneros Meliola, Irenopsis y Asteridiella no se consideran recurrentes, ya que por su relación trófica siempre estarán asociadas a sus hospederos.

Finalmente, para poder tener una idea más clara de las relaciones ecológicas de los ascomycetes en el BMM, es necesario: 1) intensificar las colectas, 2) determinar su grupo trófico y en qué sustratos crecen; sobre esto último la búsqueda bibliográfica evidenció que es raro que los colectores identifiquemos hasta género o especie los árboles a los que se asocian los hongos, 3) identificar si están asociados a una estructura particular de su hospedero (tronco, rama, hojas, fruto, madera en descomposición). La carencia de esta información impide inferir con certeza si un hospedero determinado puede albergar la misma comunidad de hongos en regiones diferentes. También se carece de datos en México sobre si existe alguna especificidad entre los hongos (en particular los saprobios) y la categoría taxonómica de la planta (familia, género o especie). Tomar en cuenta todo lo anterior nos permitiría avanzar mucho sobre el conocimiento de la relación planta-hongo dentro del BMM.

\section{AGRADECIMIENTOS}

Al Dr. Gastón Guzmán por su invaluable ayuda en la consulta de la colección de hongos del herbario XAL del Instituto de Ecología, al técnico de la misma Juan Lara Carmona por su apoyo en el trabajo de herbario, y especialmente al Dr. Fran- 
cisco Lorea por sus atinados comentarios y ayuda en la información botánica. Al Biól. Ranulfo Castillo se le agradece la elaboración de las tablas y la edición de las gráficas y figuras.

\section{LITERATURA CITADA}

Bautista, N., S. Chacón y G. Guzmán. 1986. Ascomycetes poco conocidos de México, III. Especies del estado de Morelos. Rev. Mex. Mic. 2: 85-104.

Bubbs, P., I. May, L. Miles y J. Sayer. 2004 Cloud forest agenda. United Nations Environment Programme - World Conservation Monitoring Centre. Cambridge, UK. En línea http://www.unep-wcmc.org/medialibrary/2010/09/10/a7c88b36/Cloud_Forest.pdf

Calderón de Rzedowski, G. 1979. Alchemilla. In: Rzedowski, J. y G. Calderón de Rzedowski (eds.). Flora fanerogámica del Valle de México. Vol 1. editorial CECSA. México, D.F., México. pp. 262-265.

Carrión, G. y S. Chacón. 1984. Nuevos registros de Ascomycetes fitopatógenos en México. Bol. Soc. Mex. Mic. 19: 193-199.

Carrión, G. y S. Chacón. 1985. Primer registro en México de Botryosphaeria ribis y Mammiania fimbriata (Ascomycetes). Rev. Mex. Mic. 1: 345-348.

Carrión, G. y S. Chacón. 1993. Primer registro de Balansia cyperi (fungi) en México. Rev. Mex. Mic. 9: 165-167.

Cibrián T. D., D. Alvarado y S. E. García. 2007. Enfermedades forestales en México/ Forest Diseases in Mexico. Universidad Autónoma de Chapingo. Chapingo, México. 587 pp.

Chacón, S. y F. Cruz. 1999. Descripción de 13 nuevos registros de mildiús negros (Meliolales) del estado de Veracruz, México. Rev. Mex. Mic. 15: 23-36.

Chacón, S. y G. Guzmán. 1983a. Penzigia conostoma y Penzigia enteroleuca (Ascomycetes, Pyrenomycetes, Sphaeriales) en México. Bol. Soc. Mex. Mic. 18: 29-32.

Chacón, S. y G. Guzmán. 1983b. Ascomycetes poco conocidos de México. Bol. Soc. Mex. Mic. 18: 183-218.

Chacón, S. y R. Medel. 1988. Ascomycetes lignícolas de México I. Diatrypales. Rev. Mex. Mic. 4: 323-331.

Chacón, S. y R. Medel. 1992. Ascomycetes poco conocidos de México VI. Algunos Discomycetes y Pyrenomycetes. Rev. Mex. Mic. 8: 55-62.

Chacón, S. y R. Medel. 1993. Los hongos (principalmente macromicetos) registrados en el bosque mesófilo de montaña de México. In: Marmolejo, J. y F. Garza-Ocañas. Contribuciones micológicas en homenaje al Biólogo José Castillo Tovar, por su labor en pro de la micología mexicana. Número Especial 13. Facultad de Ciencias Forestales, Universidad Autónoma de Nuevo León. Linares, México. pp. 61-110.

Challenger, A. 1998. Utilización y conservación de los ecosistemas terrestres de México. Pasado, presente y futuro. Comisión Nacional para el Conocimiento y Uso de la Biodiversidad, Instituto de Biología, Universidad Nacional Autónoma de México y Agrupación Sierra Madre. México, D.F. México. 847 pp. 
Challenger, A. y J. Soberón. 2008. Los ecosistemas terrestres. In: Capital natural de México, Vol. 1: Conocimiento actual de la biodiversidad. Comisión Nacional para el Conocimiento y Uso de la Biodiversidad. México, D.F., México. pp. 87-108.

Díaz-Barriga, H. y S. Chacón. 1992. Nuevo registro del género Microglossum (Ascomycotina, Geoglossaceae) para la micoflora mexicana. Acta Bot. Mex. 20: 5-8.

García, J. y A. López. 1993. Podosordaria mexicana y P. leporina. Centro de Genética Forestal, Universidad Veracruzana. Notas Técnicas No. 7: 1-2.

Guzmán, G. y F. Tapia. 1998. The known morels in Mexico, a description of a new blushing species, Morchella rufobrunnea and new data on M. guatemalensis. Mycologia 90: 705-714.

Guzmán, G., M. A. Morón, F. Ramírez-Guillén y J. H. D. Wolf. 2001. Entomogenous Cordyceps and related genera from Mexico with discussions on their hosts and new records. Mycotaxon 78: 115-125.

Haines, J. H. 1980. Studies in the Hyaloscyphaceae I. Some species of Dasyscyphus on tropical ferns. Mycotaxon 11: 189-216.

Heredia, G. 1989. Estudio de los hongos de la Reserva de la Biosfera El Cielo, Tamaulipas. Consideraciones sobre la distribución y ecología de algunas especies. Acta Bot. Mex. 7: 1-18.

Hyde, K. D., B. Bussaban, B. Paulus, P. W. Crous, S. Lee, E. H. C. Mckenzie, W. Photita y S. Lumyong. 2007. Diversity of saprobic microfungi. Biodiv. Conserv. 16: 7-35.

Johnston, F. R. y D. W. Minter. 1989. Structure and taxonomic significance of the ascus in the Coryneliaceae. Myc. Res. 92(4): 422-430.

Ju, Y.-M. y J. D. Rogers. 1996. A revision of the genus Hypoxylon. Mycologia Memoirs 20, American Phytopathological Society Press. St. Paul, Minnesota, USA. 365 pp.

Ju, M-Y., J. D. Rogers y F. San Martin. 1997. A revision of the genus Daldinia. Mycotaxon 61: 243-293.

Kuo, M. 2008. Morchella tomentosa, a new species from western North America, and notes on M. rufobrunnea. Mycotaxon 105: 441-446.

López, A. y J. García. 2001a. Orbilia sarraziniana. Funga Veracruzana 40: 1-2. http:// fungavera.com/fungavera/funga\%20pdf/40_Orbilia\% 20sarraziniana. pdf

López, A. y J. García. 2001b. Trichophaea boudieri. Funga Veracruzana 37: 1-2. fungavera. com/fungavera/funga\%20pdf/37_Trichophaea.pdf

López, A. y J. García. 2002. Stictis radiata Funga Veracruzana 67: 1-2 http://fungavera.com/ fungavera/funga $\% 20 \mathrm{pdf} / 67$ Stictis radiata.pdf

López, A. y J. García. 2010. Hyalorbilia inflatula. Funga Veracruzana 106: 1-4.http:// fungavera.com/fungavera/funga\%20pdf/106-HYALORINFLA. pdf

Medel, R. 2007a. Ascomycetes citados de México IV: 1996-2006. Rev. Mex. Mic. 25: 69-76.

Medel, R. 2007b. First record of the genus Byssosphaeria (Pleosporales) in Mexico. Mycotaxon 100: 247-250.

Medel, R., y F. D. Calonge. 2004. Aportación al conocimiento de los discomicetes de México, con especial referencia al género Helvella. Bol. Soc. Micol. Madrid 28: 151- 159.

Medel, R., F. D. Calonge y G. Guzmán. 2006. Nuevos registros de Pezizales (Ascomycota) de Veracruz. Rev. Mex. Mic. 23: 83-86.

Medel, R. y F. Lorea-Hernández. 2008. Hyaloscyphaceae growing on tree ferns. Mycotaxon 106: 209-217. 
Medel, R., G. Guzmán y S. Chacón. 1995. New data on the genus Wynnea in Mexico. Mycotaxon 55: 295-299.

Medel, R., G. Guzmán, S. Chacón y R. P. Korf. 1996. Iodowynnea, a new genus of the Pezizales known from Africa and tropical America. Mycotaxon 59: 127-135.

Medel, R., G. Guzmán y S. Chacón. 1999. Especies de macromicetos citadas de México. IX. Ascomycetes parte III: 1983-1996. Acta Bot. Mex. 46: 57-72.

Medel, R., G. Guzmán y R. Castillo. 2008. Las especies de Xylaria (Ascomycota, Xylariaceae) conocidas de Veracruz, México y discusión de nuevos registros. Rev. Mex. Mic. 28: 101-118.

Medel, R. y S. Chacón. 1988a. Primer registro en México de Patinellaria cubensis (Discomycetes, Helotiales) y su asociación con Xylaria arbuscula. Rev. Mex. Mic. 4: 251-254.

Medel, R. y S. Chacón. 1988b. Ascomycetes lignícolas de México, II. Algunos Pyrenomycetes y Discomycetes. Mic. Neotrop. Aplic. 1: 87-96.

Medel, R. y S. Chacón. 1992. Ascomycetes lignícolas de México III. Algunos Sphaeriales. Int. J. Mycol \& Lichenol. 5: 253-260.

Medel, R. y S. Chacón. 1997. Ascomycetes poco conocidos de México VIII. Algunas especies del bosque mesófilo de Veracruz. Acta Bot. Mex. 39: 43-52.

Medel, R. y S. Chacón. 2000. Contribución al conocimiento del género Plectania (Pezizales, Sarcosomataceae) en México. Acta Bot. Mex. 50: 11-19.

Medel, R., S. Chacón y G. Guzmán. 1989. Especies conocidas y nuevos registros de Hypoxylon (Sphaeriales, Xylariaceae) en México. Rev. Mex. Mic. 5: 149-168.

Nee, M. 1993. Solanaceae II (Solanum). Flora de Veracruz 72: 1-158.

Pérez-Silva, E. 1978. Nuevos registros del género Cordyceps (Pyrenomycetes) en México. Bol. Soc. Mex. Mic. 12: 19-25.

Pérez-Silva, E. 1983. Distribución de algunas especies del género Hypoxylon (Pyrenomycetes) en México. An. Inst. Biol. Univ. Nac. Aut. Méx., Ser. Bot. 54: 1-22.

Pérez-Silva, E. Aguirre-Acosta y T. Herrera. 1983. Distribución e importancia de algunas especies de Hypomyces (Hypocreales) en México. An. Inst. Biol. Univ. Nac. Aut. Méx.,, Ser. Bot. 54: 203-218.

Pohl, R. W. 1994. Setaria. In: Davidse, G., M. Sousa y A. O. Chater (eds.). Flora Mesoamericana. Vol 6. Universidad Nacional Autónoma de México. México, D.F., México. pp. 359-363.

Pompa-González, A. y J. Cifuentes. 1991. Estudio taxonómico de los Pezizales de los estados de Guerrero, Hidalgo, Estado de México y Michoacán. Rev. Mex. Mic. 7: 87-112.

Rinaldi, A. C., O. Commandini y T. W. Kuyper. 2008. Ectomycorrihizal fungal diversity separating the wheat from the chaff. Fung. Div. 33: 1-45.

Rodríguez, O., L. Guzmán-Dávalos y L. S. Vásquez. 1993. Nuevos registros de hongos para México. Micol. Neotrop. Apl. 6: 61-71.

Rogers, J. D., F. San Martín y Y.-M. Ju. 2002. A reassessment of the Xylaria on Liquidambar fruits and two new taxa on Magnolia fruits. Sydowia 54: 91-97.

Rzedowski, J. 1978. Vegetación de México. Ed. Limusa. México, D.F., México. 432 pp.

Rzedowski, J. 1996. Análisis preliminar de la flora vascular del bosque mesófilo de montaña en México. Acta Bot. Mex. 35: 25-44. 
Samuels, G. J., C. Rogerson. 1990. Some Ascomycetes (fungi) occurring on tropical ferns. Brittonia 42(2): 105-115.

Sánchez-Jacóme, M. R. y L. Guzmán-Dávalos. 2005. New records of Ascomycetes from Jalisco, México. Mycotaxon 92: 177-191.

Sánchez, M. y G. Carrión. 1992. Nuevos registros de mildiús negros (Meliolales). Rev. Mex. Mic. 8: 27-42.

San Martín, F. y J. D. Rogers. 1989. A preliminary account of Xylaria of Mexico. Mycotaxon 34: 283-374.

San Martín, F. y J. D. Rogers. 1993. Biscogniauxia and Camillea in Mexico. Mycotaxon 47: 229-258.

San Martín, F. y J. D. Rogers. 1995. Notas sobre la historia y relaciones de hospedante y distribución del género Xylaria (Pyrenomycetes, Sphaeriales) en México. Acta Bot. Mex. 30: 21-40.

San Martín, F. y J. D. Rogers. 1996. A preliminary list of Xylariaceous fungi at El Cielo Biosphere Reserve, in Tamaulipas, Mexico. Acta Bot. Mex. 37: 23-31.

San Martín, F., J. D. Rogers y P. Lavín. 1997. Algunas especies de Xylaria (Pyrenomycetes, Sphaeriales) habitantes en hojarasca de bosques mexicanos. Rev. Mex. Mic. 13: 5869.

San Martín F., Y.-M. Ju y J. D. Rogers. 1999. Algunas especies de Hypoxylon (Pyrenomycetes, Xylariaceae) en México. Acta Bot. Mex. 47: 31-53.

San Martín, F., J. D. Rogers y P. Lavín. 2001. Some species of Xylaria (Hymenosmycetes, Xylariaceae) associated with oak. Mycotaxon 79: 337-360.

San Martín, F. y P. Lavín. 1997. Los Ascomycetes Acantonitschkea, Corynelia, Lopadostoma y Camarops en México. Acta Bot. Mex. 41: 31-41.

Standley, P. C. y L. O. Williams. 1973. Hyptis. Flora of Guatemala. Fieldiana Bot. 24 (9): 246-259.

Welden, A. L. y P. Lemke. 1961. Notas sobre algunos hongos mexicanos. Bol. Soc. Bot. Méx. 26: 1-24.

Williams-Linera, G., R. Manson y E. Isunza. 2002. La fragmentación del bosque mesófilo de montaña y patrones de uso del suelo en la región oeste de Xalapa, Veracruz, México. Madera y Bosques 8(1): 73-89.

Williams-Linera, G. 2007. El bosque de niebla del centro de Veracruz: Ecología, historia y destino en tiempos de fragmentación y cambio climático. Comisión Nacional para el Conocimiento y Uso de la Biodiversidad - Instituto de Ecología, A.C. Xalapa, Veracruz, México. 208 pp. 


\section{APÉNDICE}

Especies de Ascomycetes citados del BMM en México.Notación: Sustrato: L = lignícola $; \mathrm{E}=$ entomopatógeno; $\mathrm{Fi}$ = fimícola $; \mathrm{F}=$ folícola $; \mathrm{Fu}=$ fungícola $; \mathrm{Fr}=$ frutícola; $\mathrm{H}=$ humícola; $\mathrm{T}=$ terrícola. Grupos tróficos: $\mathrm{P}=$ parásito; $\mathrm{S}=$ saprobio; $\mathrm{SM}=$ Simbionte micorrízico.

\begin{tabular}{ccc}
\hline Especies/sustrato/grupo trófico & $\begin{array}{c}\text { Distribución } \\
\text { geográfica conocida } \\
\text { en México }\end{array}$ & Referencia bibliográfica \\
\hline
\end{tabular}

\section{Botryosphaeriales}

Botryosphaeriaceae

Botryosphaeria ribis Grossenb. \&

Duggar (L,S)

Lasiodiplodia theobromae (Pat.)

Griffon \& Maubl. (L,P)

\section{Capnodiales}

Mycosphaerellaceae

Pseudocercospora liquidambaricola

(J.M. Yen) U. Braun (F,P)

Ver.

Carrión y Chacón, 1985

Ver. Medel y Chacón, 1988b

\section{Coronophorales}

Nitschkiaceae

D.F., Edo. Méx., Cibrian et al., 2007 Hgo., Pue.

Nitschkia grevillei (Rehm) Nannf. (L,S)

\section{Coryneliales}

Coryneliaceae

Corynelia oreophila (Speg.) Starbäck

$$
\text { (L,S) }
$$

Pue., Tamps.

Ver.

Medel y Chacón, 1988b

\section{Diaporthales}

Gnomoniaceae

Gnomonia fimbriata (Pers.) Fuckel (F,S)

Melogrammataceae

Melogramma campylosporum Fr. (L,S)

\section{Dothideales}

Dimeriella polypodii Samuels \& Rogerson (L,S)

\section{Erysiphales}

Erysiphaceae

Sphaerotheca lanestris Harkn. (L,P)
Ver.

Carrión y Chacón, 1985

Ver.

Medel y Chacón, 1988b

Oax.

Samuels y Rogerson, 1990 
Apéndice. Continuación.

\begin{tabular}{lcc}
\hline Especies/sustrato/grupo trófico & $\begin{array}{c}\text { Distribución } \\
\text { geográfica conocida } \\
\text { en México }\end{array}$ \\
\hline
\end{tabular}

\section{Helotiales}

Helotiaceae

Belonioscypha sessilis K.S. Thind \& H. Singh (L,S)

Bisporella citrina (Batsch) Korf \& S.E. Carp.(L,S)

B. sulfurina (Quél.) S.E. Carp. (L,S)

Crocicreas sessilis Samuels \&

Rogerson (L,S)

Patinellaria cubensis (Berk. \& M.A.

Curt.) Dennis (Fu,S)

Pocillum cesatii (Mont.) De Not. (L,S)

Geoglossaceae

Geoglossum atropurpureum (Batch)

Pers. (H,S)

Microglossum olivaceum (Pers.) Gillet.

(H,S)

Mor. Chacón y Guzmán, 1983b

Ver. $\quad$ Medel y Calonge, 2004

Mor. Chacón y Guzmán, 1983b

Oax. Samuels y Rogerson, 1990

Ver. Medel y Chacón, 1988a

Ver. $\quad$ Medel y Chacón, 1992

Ver. Medel y Calonge, 2004

Hyaloscyphaceae

Lachnum brasiliense (Mont.) Haines \& Dumont (L,S)

L. corticola (Massee) M.P. Sharma

\section{(L,S)}

L. fimbriiferum (Berk. \& M.A. Curtis) J.H. Haines $(\mathbf{L}, \mathbf{S})$

L. oncospermatum (Berk. \& Broome)

M.L. Wu \& J.H. Haines (L,S)

L. pteridophyllum (Rodway) Spooner (L,S)

L. singerianum (Dennis) W.Y. Zhuang \& Zheng Wang (L,S)

L. soppitii (Massee) Raitv. (L,S)

L. varians (Rehm) M.P. Sharma (L,S)

Dermataceae

Belonopsis obscura (Rehm) Aebi (L,S)

Mor., Ver. Chacón y Guzmán, 1983b

Edo. Méx. Ver. Chacón y Guzmán, 1983b;

Welden y Lemke, 1961

Ver. Medel y Lorea-

Hernández, 2008

Ver. Medel y Lorea-

Hernández, 2008

Oax., Ver. Haines, 1980; Medel y

Lorea-Hernández, 2008

Ver. Medel y Lorea-

Hernández, 2008

Ver. $\quad$ Medel y Calonge, 2004

Chis., Ver. Medel y Lorea-

Hernández, 2008

Mor. Chacón y Guzmán, 1983b 
Apéndice. Continuación.

\begin{tabular}{|c|c|c|}
\hline Especies/sustrato/grupo trófico & $\begin{array}{l}\text { Distribución } \\
\text { geográfica conocida } \\
\text { en México }\end{array}$ & Referencia bibliográfica \\
\hline $\begin{array}{l}\text { Mollisia undulatodepressula (Feltgen) } \\
\text { Le Gal \& F. Mangenot }(\mathbf{L}, \mathbf{S})\end{array}$ & Ver. & Medel y Chacón, 1997 \\
\hline \multicolumn{3}{|l|}{ Hypocreales } \\
\hline \multicolumn{3}{|l|}{ Clavicipitaceae } \\
\hline Balansia cyperi Edgerton (F,P) & Ver. & Carrión y Chacón, 1993 \\
\hline \multicolumn{3}{|l|}{ Cordycipitaceae } \\
\hline $\begin{array}{l}\text { Ophiocordyceps dipterigena (Berk. } \\
\text { \& Broome) G.H. Sung, J.M. Sung, } \\
\text { Hywel-Jones \& Spatafora (E,P) }\end{array}$ & Ver. & Guzmán et al., 2001 \\
\hline $\begin{array}{l}\text { O. gracilis (Grev.) G.H. Sung, J.M. } \\
\text { Sung, Hywel-Jones \& Spatafora (E,P) }\end{array}$ & Jal. & Rodríguez et al., 1993 \\
\hline $\begin{array}{l}\text { O. entomorrhiza (Dicks.) G.H. Sung, } \\
\text { J.M. Sung, Hywel-Jones \& Spatafora } \\
\text { (E,P) }\end{array}$ & Ver. & Pérez-Silva, 1978 \\
\hline \multicolumn{3}{|l|}{ Hypocreaceae } \\
\hline $\begin{array}{l}\text { Hypomyces. luteovirens (Fr.) Tul. \& C. } \\
\text { Tul. (Fu,P) }\end{array}$ & Edo. Méx., Mor. & Pérez-Silva et al., 1983 \\
\hline \multicolumn{3}{|l|}{ Leotiales } \\
\hline \multicolumn{3}{|l|}{ Leotiaceae } \\
\hline Bulgaria inquinans (Pers.) Fr. (L,S) & Mor. & Bautista et al., 1986 \\
\hline \multicolumn{3}{|l|}{ Meliolales } \\
\hline \multicolumn{3}{|l|}{ Meliolaceae } \\
\hline $\begin{array}{l}\text { Appendiculella calostroma (Desm.) } \\
\text { Höhn. (F,P) }\end{array}$ & Ver. & Sánchez y Carrión, 1992 \\
\hline $\begin{array}{l}\text { Asteridiella anastomosans (G. Winter) } \\
\text { Hansf. (F,P) }\end{array}$ & Ver. & Sánchez y Carrión, 1992 \\
\hline $\begin{array}{l}\text { A. melastomatacearum (Speg.) Hansf. } \\
(\mathbf{F}, \mathbf{P})\end{array}$ & Ver. & Sánchez y Carrión, 1992 \\
\hline A. toroana (Cif.) Hansf. (F,P) & Ver. & Sánchez y Carrión, 1992 \\
\hline Irenopsis costaricensis F. Stev. (F,P) & Ver. & Chacón y Cruz, 1999 \\
\hline I. tortuosa (Wint.) Stevens (F,P) & Ver. & Sánchez y Carrión, 1992 \\
\hline Meliola ambigua Pat. \& Gaillard (F,P) & Ver. & Sánchez y Carrión, 1992 \\
\hline $\begin{array}{l}\text { M. agavicola Mig. Rodr. \& Camino } \\
\text { (F,P) }\end{array}$ & Ver. & Chacón y Cruz, 1999 \\
\hline M. malacotricha Speg. (F,P) & Ver. & Sánchez y Carrión, 1992 \\
\hline
\end{tabular}


Apéndice. Continuación.

\begin{tabular}{|c|c|c|}
\hline Especies/sustrato/grupo trófico & $\begin{array}{c}\text { Distribución } \\
\text { geográfica conocida } \\
\text { en México }\end{array}$ & Referencia bibliográfica \\
\hline M. mimosacearum Hansf. (F,P) & Ver. & Sánchez y Carrión, 1992 \\
\hline $\begin{array}{l}\text { M. panici var. panicicola (Syd.) Hansf. } \\
\text { (F,P) }\end{array}$ & Ver. & Chacón y Cruz, 1999 \\
\hline M. trichiliae Beeli (F,P) & Ver. & Chacón y Cruz, 1999 \\
\hline M. weismarensis F. Stevens (F,P) & Ver. & Sánchez y Carrión, 1992 \\
\hline
\end{tabular}

\section{Orbiliales}

Orbiliaceae

Hyalorbilia inflatula (P. Karst.) Baral \&

G. Marson (L,S)

Orbilia sarraziniana Boud. (L,S)

Ver. López y García, 2010

Ostropales

Ver. López y García, 2001a

Stictidaceae

Stictis radiata (L.) Pers. (L,S)

Ver.

López y García, 2002

Pezizales

Helvellaceae

Helvella brevis (Peck) Harmaja (T,SM)

Ver. $\quad$ Medel y Calonge, 2004

H. queletii Bres. (T,SM)

Ver. Chacón y Medel, 1992

H. stevensii Peck (T,SM)

Mor. Bautista et al., 1986

Morchellaceae

Morchella rufobrunnea Guzmán \&

Ver.

Guzmán y Tapia, 1998

Tapia (T,S)

Pezizaceae

Iodowynnea auriformis (Pat. ex Le Gal)

Medel, Guzmán \& Chacón (T,S)

Peziza ampliata Pers. (T,SM)

Ver. $\quad$ Medel et al., 1996

P. succosella (Le Gal \& Romagn.)

M.M. Moser ex Aviz Hersh \&

Nemlich (T,SM)

Pyronemataceae

Humaria leucoloma (Hedw.) Sacc.

(T,SM)

Paratrichophaea boudieri (Grelet)

Bronckers (H,S)

Jal. Sánchez-Jácome y

Guzmán-Dávalos, 2005

Ver. Medel et al., 2006

Ver. Chacón y Medel, 1992

Ver. López y García, 2001b 
Apéndice. Continuación.

\begin{tabular}{lcc}
\hline Especies/sustrato/grupo trófico & $\begin{array}{c}\text { Distribución } \\
\text { geográfica conocida } \\
\text { en México }\end{array}$ & Referencia bibliográfica \\
\hline
\end{tabular}

Sarcosomataceae

Plectania campylospora (Berk.) Nannf.

$$
\text { (L,S) }
$$

P. rhytidia f. rhytidia (Berk.) Nannf. \& Korf (L,S)

P. rhytidia f. platensis (Speg.) Donadini

$$
\text { (L,S) }
$$

Sarcoscyphaceae

Wynnea macrotis (Berk.) Berk. (T,S)

\section{Pleosporales}

Melanommataceae

Byssosphaeria jamaicana (Sivan.)

M.E. Barr (L,S)

\section{Rhytismatales}

Rhytismataceae

Propolis farinosa (Pers.) Fr. (L,S)

\section{Sordariales}

Lasiosphaeriaceae

Lasiosphaeria hispida (Tode) Fuckel

$$
\text { (L,S) }
$$

Xylariales

Diatrypaceae

Diatrype albopruinosa (Schwein.)

Cooke (L,S)

D. capnostoma Berk. \& Ravenel (L,S)

Xylariaceae

Biscogniauxia mediterranea (De Not.) Kuntze (L,P)

Daldinia childiae J.D. Rogers \& Y.-M. $\mathrm{Ju}$ (L,P)

D. clavata Henn. (L,P)

D. cudonia (Berk. \& M.A. Curtis)

$$
\text { Lloyd (L,P) }
$$

Hypoxylon aeruginosum var. macrosporum J.D. Rogers (L,S)
Gro. Pompa-González y Cifuentes, 1991

Ver. Medel y Chacón, 2000

Ver. Medel y Chacón, 2000

Ver. $\quad$ Medel et al., 1995

Ver. $\quad$ Medel, 2007b

Ver. $\quad$ Medel y Chacón, 1997

Ver. $\quad$ Medel y Chacón, 1997

Ver. Chacón y Medel, 1988

Qro. Chacón y Medel, 1988

N.L., Tamps. San Martín y Rogers, 1993

Tamps. Ju et al., 1997

Tamps. Ju et al., 1997

Ver. Ju et al., 1997

Ver. Chacón y Medel, 1992 
Apéndice. Continuación.

\begin{tabular}{|c|c|c|}
\hline Especies/sustrato/grupo trófico & $\begin{array}{c}\text { Distribución } \\
\text { geográfica conocida } \\
\text { en México }\end{array}$ & Referencia bibliográfica \\
\hline $\begin{array}{l}\text { H. bovei var. microsporum J.H. Mill. } \\
\text { (L,S) }\end{array}$ & Ver. & Pérez-Silva, 1983 \\
\hline H. fuscum (Pers.) Fr. (L,S) & Chis. & Pérez-Silva, 1983 \\
\hline H. notatum Berk. \& M.A. Curtis (L,S) & Ver. & Medel et al., 1989 \\
\hline $\begin{array}{l}\text { H. punctulatum (Berk. \& Ravenel) } \\
\text { Cooke (L,S) }\end{array}$ & Ver. & Medel et al., 1989 \\
\hline $\begin{array}{l}\text { H. shearii Y.-M. Ju \& J.D. Rogers } \\
\text { (L,S) }\end{array}$ & Tamps. & San Martín et al., 1999 \\
\hline H. subgilvum Berk. \& Broome (L,S) & Tamps. & Ju y Rogers, 1996 \\
\hline H. subrutilum Starbäck (L,S) & Tamps. & Ju y Rogers, 1996 \\
\hline H. aff. ticinense L.E. Petrini (L,S) & Tamps. & San Martin et al., 1999 \\
\hline $\begin{array}{l}\text { Kretzschmariella culmorum (Cooke) } \\
\text { Y.M. Ju \& J.D. Rogers (L,S) }\end{array}$ & Qro. & Medel et al., 1989 \\
\hline $\begin{array}{l}\text { Nemania bipapillata (Berk. \& M.A. } \\
\text { Curtis) Pouzar (L,S) }\end{array}$ & Qro. & Medel et al., 1989 \\
\hline N. effusa (Nitschke) Pouzar (L,S) & Tamps. & San Martín y Rogers, 1996 \\
\hline $\begin{array}{l}\text { Penzigia berteroi (Mont.) L.W. Mill. } \\
\quad(\mathbf{L}, \mathbf{S})\end{array}$ & Ver. & Medel y Chacón, 1997 \\
\hline P. conostoma (Mont.) J. H.Mill.(L,S) & Ver. & Chacón y Guzmán, 1983a \\
\hline $\begin{array}{l}\text { Podosordaria leporina (Ellis \& Everh.) } \\
\text { Dennis (Fi,S) }\end{array}$ & Ver. & Medel y Chacón, 1992 \\
\hline P. mexicana Ellis \& Holw. (Fi,S) & Ver. & García y López, 1993 \\
\hline $\begin{array}{l}\text { Thuemenella cubispora (Ellis \& Holw.) } \\
\text { Boedijn (L,S) }\end{array}$ & Mor. & Chacón y Guzmán, 1983b \\
\hline Xylaria aenea Mont. (L,S) & Chis. & $\begin{array}{l}\text { San Martín y Rogers, } \\
1995\end{array}$ \\
\hline X. adscendens (Fr.) Fr. (L,S) & Tamps., Ver. & $\begin{array}{l}\text { San Martin y Rogers, } \\
\text { 1989; San Martin et al., } \\
\text { 2001; Medel et al., } 2008\end{array}$ \\
\hline X. apiculata Cooke (L,S) & Chis., Tamps. & $\begin{array}{l}\text { San Martin y Rogers, } \\
1995\end{array}$ \\
\hline$X$. arbuscula Sacc. (L,S) & Tamps., Ver. & $\begin{array}{l}\text { Medel y Chacón, 1988a } \\
\text { San Martin y Rogers, } \\
1989\end{array}$ \\
\hline X. corniculata Sacc. $\mathbf{( L , S )}$ & Ver. & $\begin{array}{l}\text { San Martin y Rogers, } \\
\text { 1989; Medel et al., } 2008\end{array}$ \\
\hline
\end{tabular}


Apéndice. Continuación.

\begin{tabular}{|c|c|c|}
\hline Especies/sustrato/grupo trófico & $\begin{array}{c}\text { Distribución } \\
\text { geográfica conocida } \\
\text { en México }\end{array}$ & Referencia bibliográfica \\
\hline $\begin{array}{l}\text { X. enteroleuca (Speg.) P.M.D. Martín } \\
(\mathbf{L}, \mathbf{S})\end{array}$ & Ver. & Chacón y Guzmán, 1983a \\
\hline $\begin{array}{l}\text { X. eugeniae San Martín, Vanoye \& P. } \\
\text { Lavín (F,S) }\end{array}$ & Tamps. & San Martín et al., 1997 \\
\hline $\begin{array}{l}\text { X. jaliscoensis San Martín, J.D. Rogers } \\
\quad \& \text { Y.-M. Ju (L,S) }\end{array}$ & Jal. & Rogers et al., 2002 \\
\hline $\begin{array}{l}\text { X. liquidambaris J.D. Rogers, Y.M Ju } \\
\text { \& F. San Martín, (Fr,S) }\end{array}$ & Ver. & $\begin{array}{l}\text { Rogers et al., } 2002 \text { Medel } \\
\text { et al., } 2008\end{array}$ \\
\hline$X$. longipes Nitschke $(\mathbf{L}, \mathbf{S})$ & Jal., Tamps. & $\begin{array}{l}\text { San Martin y Rogers, } \\
1995\end{array}$ \\
\hline X. magnoliae J.D.Rogers (Fr,S) & Tamps. & Heredia, 1989 \\
\hline X. melanura (Lév.) Sacc. (L,S) & Chis. & $\begin{array}{l}\text { San Martín y Rogers, } \\
1995\end{array}$ \\
\hline $\begin{array}{l}\text { X. michoacana San Martín, J.D. Rogers } \\
\text { \& P. Lavín }(\mathbf{L}, \mathbf{S})\end{array}$ & Mich. & San Martín et al., 2001 \\
\hline X. microceras (Mont.) Berk. (L,S) & Ver. & Medel et al., 2008 \\
\hline $\begin{array}{l}\text { X. persicaria (Schwein.) Berk. \& M.A. } \\
\text { Curtis (Fr,S) }\end{array}$ & Tamps., Ver. & $\begin{array}{l}\text { San Martín y Rogers, } \\
1989,1995\end{array}$ \\
\hline X. phosphorea Berk. (L,S) & Tamps. & $\begin{array}{l}\text { San Martín y Rogers, } \\
1995\end{array}$ \\
\hline $\begin{array}{l}\text { X. subcoccophora San Martín \& P. } \\
\text { Lavín }(\mathbf{L}, \mathbf{S})\end{array}$ & Tamps. & San Martín et al., 2001 \\
\hline $\begin{array}{l}\text { X. tumulosa San Martín, J.D. Rogers \& } \\
\quad \text { P. Lavín }(\mathbf{L}, \mathbf{S})\end{array}$ & Chis. & San Martín et al., 2001 \\
\hline
\end{tabular}

